\title{
Plasma application in aesthetic medicine: Clinical and physical aspects
}

\author{
Mohsen Pourazizi ${ }^{1,2}$, Bahareh Abtahi-Naeini ${ }^{3 *}$ \\ ${ }^{1}$ Cancer Research Center (CRC), Semnan University of Medical Sciences, Semnan, Iran \\ ${ }^{2}$ Isfahan Eye Research Center (IERC), Isfahan University of Medical Sciences, Isfahan, Iran \\ ${ }^{3}$ Skin Diseases and Leishmaniasis Research Center, Isfahan University of Medical Sciences, Isfahan, Iran
}

Keywords: Plasma technology; plasma skin regeneration, aesthetic, rejuvenation

Citation: Pourazizi M and Abtahi-Naeini B. Plasma application in aesthetic medicine: Clinical and physical aspects. J Surg Dermatol 2017; 2(T1): 113-114; http://dx.doi.org/10.18282/jsd.v2.it1.140.

*Correspondence to: Bahareh Abtahi-Naeini, Skin Diseases and Leishmaniasis Research Center, Isfahan University of Medical Sciences, Isfahan, Iran, Bahareh.abtahi@yahoo.com.

Received: $21^{\text {st }}$ February 2017; Published Online: $15^{\text {th }}$ March 2017

\section{Introduction}

Aesthetic outcome in patients' demand for cosmetic treatments have been influenced by some parameters such as clinical improvement after procedures, risk and potential complications, and downtime periods ${ }^{[1,2]}$. These parameters could validate plasma technology as a new regenerative modality. A basic understanding of plasma physics and histopathological aspects of plasma is required to understand the impact of plasma technology on aesthetic medicine.

\section{Physical aspects of plasma}

Plasma is the fourth state of matter, which comprises ionized atoms ${ }^{[1,3]}$. Almost the entire universe comprises this matter ${ }^{[4]}$. When sufficient energy is delivered to gas atoms, ionization occurs and the atoms acquire a positive charge after the electrons escape ${ }^{[1,3]}$.

In terms of pressure, plasma can be categorized into low-pressure, atmospheric-pressure, and high-pressure plasma. Furthermore, in terms of relative temperatures, it can be classified into thermal and non-thermal plas$\mathrm{ma}^{[4]}$. Plasma can also be produced artificially.

For artificial plasma production, using an ul- tra-high-frequency radiofrequency generator, the energy applied to the gas atoms is emitted in a millisecond pulse to the skin via a handpiece ${ }^{[5]}$. Thermal-air-based electrical discharge plasma is used in cosmetic regeneration.

\section{Histopathological aspects of plasma}

Plasma action's mechanism on the skin involves two steps: immediate tissue contraction and thermal disruption. Denaturation of collagen and other proteins in the dermis following the thermal effect of plasma induces clinically observed, immediate tissue contraction ${ }^{[1]}$.

Cascade of neo-collagenization has been stimulated through thermal disruption of dermal solar elastosis, fibroblasts activation, and migration from the deeper dermis and cytokine release $\mathrm{e}^{[1,6,7]}$.

Plasma is more uniform than ablative resurfacing lasers, including carbon dioxide $\left(\mathrm{CO}_{2}\right)$ resurfacing laser, since it does not depend on the interaction with a specific target $^{[1]}$.

Regarding the level of energy of plasma and tissue shedding, high-energy plasma induces shedding in epidermis and upper dermis, whereas low-energy plasma induces shedding in only the upper part of tum corneum ${ }^{[6,7]}$. 
An experimental study on comparing the histopathological effect of plasma skin regeneration (PSR) and $\mathrm{CO}_{2}$ laser in terms of tissue and plasma interaction was conducted in $2008^{[8]}$. The results of this animal study demonstrated that high-fluence $\mathrm{CO}_{2}$ laser generated a greater thickness of thermal damage zone than any energy level of PSR. Furthermore, it revealed that plasma produced equal thermal damage zone from the low- and medium-fluence $\mathrm{CO}_{2}$ laser ${ }^{[8]}$.

Epidermal necrosis using low-energy settings PSR were less than or similar to epidermal changes in the lowest fluence in $\mathrm{CO}_{2}$ laser ${ }^{[8]}$.

\section{Clinical aspects of plasma}

PSR technology helps in the treatment of facial rhytides, solar keratosis, seborrhoeic keratosis, and warts ${ }^{[4]}$. Some studies have considered the clinical effects of PSR technology on periorbital rejuvenation ${ }^{[9,10]}$. More than $90 \%$ improvement with conventional blepharoplasty and approximately $20 \%$ and $30 \%$ improvement using PSR in the tightening of upper eyelid and peri-orbital wrinkles were achieved, respectively ${ }^{[9]}$. Approximately $40 \%$ improvement in facial acne scars after six months was achieved after a single treatment of $\mathrm{PSR}^{[8]}$.

PSR has been used in the rejuvenation of non-facial skin, including the chest, neck, and dorsum of hands ${ }^{[6]}$. Furthermore, it can be used to treat traumatic scars, benign familial pemphigus, and porokeratosis ${ }^{[1,11]}$.

Although no major side effects in clinical studies have been reported to date, side effects could occur due to the heat delivered to the skin, including erythema, edema, scaling, crusting, scarring, and skin pigmentation $^{[1]}$.

\section{Conclusion}

Plasma application has great potential in dermatology; however, further clinical and histopathological studies are required to support previous findings and to address some issues and questions about safety and efficacy.

\section{Conflict of interest}

The authors declare no potential conflict of interest with respect to the research, authorship, and/or publication of this article.

\section{References}

1. Foster KW, Moy RL, Fincher EF. Advances in plasma skin regeneration. J Cosmet Dermatol 2008; 7(3): 169-179. doi: 10.1111/j.1473-2165.2008.00385.x.

2. Ji Y, Guo L, Zhang Y, Liu Y. Comparison of topical anesthesia and subcutaneous infiltration anesthesia with regard to effect of plasma skin regeneration system. J Cosmet Laser Ther 2015; 17(6): 330-334. doi: 10.3109/14764172.2015.1027233.

3. Elsaie ML, Kammer JN. Evaluation of plasma skin regeneration technology for cutaneous remodeling. J Cosmet Dermatol 2008; 7(4): 309-311. doi: 10.1111/j.1 473-2165.2008.00411.x.

4. Heinlin J, Isbary G, Stolz W, Morfill G, Landthaler M, et al. Plasma applications in medicine with a special focus on dermatology. J Eur Acad Dermatol Venereol 2011; 25(1): 1-11. doi: 10.1111/j.1468-3083.2010.03702.x.

5. Bernstein EF. Very low energy plasma skin resurfacing treatments improve photodamage. Lasers Surg Med 2007; 39(Suppl. 19): 17.

6. Alster TS, Konda S. Plasma skin resurfacing for regeneration of neck, chest, and hands: Investigation of a novel device. Dermatol Surg 2007; 33(11): 1315-1321. doi: 10.1097/00042728-200711000-00003.

7. Bogle MA, Arndt KA, Dover JS. Evaluation of plasma skin regeneration technology in low-energy full-facial rejuvenation. Arch Dermatol 2007; 143(2): 168-174. doi: 10.1001/archderm.143.2.168.

8. Fitzpatrick R, Bernstein E, Iyer S, Brown D, Andrews P, et al. A histopathologic evaluation of the plasma skin regeneration System (PSR) versus a standard carbon dioxide resurfacing laser in an animal model. Lasers Surg Med 2008; 40(2): 93-99. doi: 10.1002/lsm.20547.

9. Biesman B. Portrait plasma blepharoplasty study and treatment results. In: Portrait plasma skin regeneration: Treatment protocols and aesthetic outcomes. American Society of Dermatologic Surgery 2007 Meeting; 2007 Oct 11-14; Chicago, Illinois.

10. Holcomb JD, Kent KJ, Rousso DE. Nitrogen plasma skin regeneration and aesthetic facial surgery: Multicenter evaluation of concurrent treatment. Arch Facial Plast Surg 2009; 11(3): 184-193. doi: 10.1001/archfacial.2009.29.

11. Kono T, Groff WF, Sakurai H, Yamaki T, Soejima K, et al. Treatment of traumatic scars using plasma skin regeneration (PSR) system. Lasers Surg Med 2009; 41(2): 128-130. doi: 10.1002/1sm.20723. 